Tropical Journal of Pharmaceutical Research April 2016; 15 (4): 821-826

ISSN: $1596-5996$ (print); 1596-9827 (electronic)

(C) Pharmacotherapy Group, Faculty of Pharmacy, University of Benin, Benin City, 300001 Nigeria.

All rights reserved.

Available online at http://www.tjpr.org

Original Research Article

http://dx.doi.org/10.4314/tjpr.v15i4.22

\title{
Synthesis and antibacterial profile of novel azomethine derivatives of $\beta$-phenylacrolein moiety
}

\author{
Sridevi Chigurupati*, Neeraj Kumar Fuloria ${ }^{1}$, Shivkanya Fuloria ${ }^{1}$, Sundram \\ Karupiah $^{1}$, Ravichandran Veerasamy ${ }^{1}$, Appala Raju Nemala ${ }^{2}$, Lim Jun Yi $^{1}$, Ang \\ xiang Ilan ${ }^{1}$ and Syed Adnan Ali Shah ${ }^{3}$ \\ ${ }^{1}$ AIMST University, Faculty of Pharmacy, Semeling, 08100 Bedong, Kedah, Malaysia, ${ }^{2}$ Department of Pharmaceutical \\ chemistry, Sultan-UI-Uloom College of Pharmacy, Road No. 3, Banjara hills, Hyderabad, India, ${ }^{3}$ Universiti Teknologi MARA \\ (UiTM), Faculty of Pharmacy, Puncak Alam Campus, Selangor D.E, Malaysia
}

*For correspondence: Email: sridevi.phd@gmail.com; Tel: +6-014-9449846

Received: 16 December 2015

Revised accepted: 24 March 2016

\begin{abstract}
Purpose: To develop some novel molecules effective against antibiotic-resistant bacterial infections. Methods: A series of azomethines (SB-1 to SB-6) were synthesized from $\beta$-phenyl acrolein moiety. The structures of the synthesized compounds were confirmed on the basis of their UV ultra-violet (UV) spectroscopy ( $\lambda_{\max }: 200$ - $400 \mathrm{~nm}$ ), Fourier transform infra-red (FTIR, vibrational frequency: 500-4000 $\mathrm{cm}^{-1}$ ), ${ }^{1} \mathrm{H}$ nuclear magnetic resonance (NMR, chemical shift: 0 - $\left.10 \mathrm{ppm}\right),{ }^{13} \mathrm{C} N \mathrm{NM}$ (chemical shift: 0 $200 \mathrm{ppm})$, mass spectrometry ( $\mathrm{m} / \mathrm{z}$ values: 0 - 500) and carbon hydrogen nitrogen $(\mathrm{CHN})$ elemental analysis. The new compounds were screened for antibacterial activity by test-tube dilution and disc diffusion methods using gentamicin as reference standard.

Results: The structures of azomethine were in full agreement with their spectral data. Among all the synthesized compounds, compounds SB-5 and SB-6 exhibited the highest minimum inhibitory concentration (MIC) of $62.5 \mu \mathrm{g} / \mathrm{mL}$. At MIC of $250 \mu \mathrm{g} / \mathrm{mL}$, all compounds SB-1 to SB-6 displayed significant antibacterial activity, compared to gentamycin $(p<0.05)$. SB-5 and SB-6 were active against $S$. aureus, $P$. aeruginosa and $K$. pneumoniae; SB-3 was active against $B$. subtilis and $S$. aureus. SB-4 was active against $P$. aeruginosa and $S$. aureus while SB-1 and SB-2 were active against $S$. aureus. Conclusion: The synthesized compounds possess antibacterial activities compared to those of gentamycin.
\end{abstract}

Keywords: Acrolein, Imines, Azomethine, Antibacterial, Gentamycin, Minimum inhibitory concentration

Tropical Journal of Pharmaceutical Research is indexed by Science Citation Index (SciSearch), Scopus, International Pharmaceutical Abstract, Chemical Abstracts, Embase, Index Copernicus, EBSCO, African Index Medicus, JournalSeek, Journal Citation Reports/Science Edition, Directory of Open Access Journals (DOAJ), African Journal Online, Bioline International, Open-J-Gate and Pharmacy Abstracts

\section{INTRODUCTION}

Antimicrobial drugs occupy a unique niche in the history of medicine. The increased incidence of severe opportunistic bacterial infections in immunological deficient patients together with the development of resistance among pathogenic Gram-positive and Gram-negative bacteria have revealed great need to search for new compounds that are effective against antibiotic- resistant bacteria. Literature highlights the potentials of $\beta$-phenyl acrolein as an antimicrobial [1], anticancer [2] and flavoring agent for chewing gums [3]. Many investigators have observed the importance of azomethines for their antibacterial [4,5] antifungal [6], antiproliferative $[7,8]$ and antipyretic properties. It is evident that azomethines with aryl substituents are more stable and readily synthesized, whereas those containing alkyl substituents are 
relatively unstable. Azomethines of aliphatic aldehydes are usually unstable and readily polymerizable, while those with aromatic aldehydes having effective conjugation are more stable [9].

In the present research work, a new series of azomethine derivatives of $\beta$-phenyl acrolein was synthesized (SB-1 to SB-6) and screened for antibacterial activity against Gram-positive (Bacillus subtilis and Staphylococcus aureus) and Gram-negative (Klebsiella pneumonia and Pseudomonas aeruginosa) bacteria in order to generate potent and safer antibacterial agents.

\section{EXPERIMENTAL}

\section{Materials}

All the solvents and chemicals used were of analytical grade and obtained from Sigma-Aldrich and Merck Pvt Ltd, India and were used without further purification.

\section{General procedure for synthesis of azomethine derivatives of $\beta$-phenyl acrolein}

Equimolar concentration of $\beta$-phenyl acrolein $(0.01 \mathrm{M})$ and substituted aromatic amines $(0.01$ M) were dissolved in $50 \mathrm{~mL}$ of anhydrous ethanol separately. Solution of substituted aromatic amine was then added drop-wise into $\beta$-phenyl acrolein solution in a conical flask. The mixture was made up to $150 \mathrm{~mL}$ with $95 \%$ anhydrous ethanol, and 2 to 3 drops of triethylamine (basic catalyst) was added [10].The mixture was then stirred using magnetic stirrer at 60 to $70^{\circ} \mathrm{C}$ for 6 $\mathrm{h}$ over a water bath. The reaction was monitored by TLC. The sample mixture was evaporated under pressure at $65{ }^{\circ} \mathrm{C}$ using rotatory evaporator [11,12]. The solid obtained on concentration of filtrate was recrystallized from aqueous ethanol to yield the pure compounds SB-1 to SB-6 (physical data are given in Table 1). The melting points of the compounds were determined on a Thoshniwal electric melting point apparatus and the values were uncorrected. The reaction was mentioned by TLC on Silica gel-GF 254 (Merck) coated plates. Spots of TLC were identified in iodine chamber.

\section{Determination of minimum inhibitory concentration (MIC)}

Test tube dilution method was used to determine minimum inhibitory concentration. One $\mathrm{mL}$ of sterilized media (Nutrient agar) was poured into sterile test tubes. One $\mathrm{mL}$ of $2000 \mu \mathrm{g} / \mathrm{mL}$ test solution was transferred in one tube and serially diluted to give concentrations of 1000, 500, 250 and $125 \mu \mathrm{g} / \mathrm{mL}$. To all the test tubes, $0.1 \mathrm{~mL}$ of suspension of bacteria in saline was added and the tubes were incubated at $37^{\circ} \mathrm{C}$ for $24 \mathrm{~h}$. The growth in the tubes was observed visually for turbidity. MIC was determined with the lowest concentration of the sample that retarded the development of turbidity [13].

Table 1: Physical data for azomethine derivatives of $\beta$ phenyl acrolein, SB-1 to SB-6

\begin{tabular}{lllc}
\hline Compound & $\begin{array}{l}\text { Molecular } \\
\text { formula }\end{array}$ & $\begin{array}{l}\text { Crystal } \\
\text { colour }\end{array}$ & Rf value \\
\hline SB-1 & $\mathrm{C}_{15} \mathrm{H}_{13} \mathrm{~N}$ & Yellow & 0.43 \\
SB-2 & $\mathrm{C}_{16} \mathrm{H}_{13} \mathrm{NO}$ & White & 0.50 \\
$\mathrm{SB}-3$ & $\mathrm{C}_{15} \mathrm{H}_{13} \mathrm{NO}$ & White & 0.56 \\
$\mathrm{SB}-4$ & $\mathrm{C}_{16} \mathrm{H}_{15} \mathrm{NO}$ & Yellow & 0.62 \\
$\mathrm{SB}-5$ & $\mathrm{C}_{17} \mathrm{H}_{15} \mathrm{NO}_{3}$ & Brown & 0.54 \\
$\mathrm{SB}-6$ & $\mathrm{C}_{16} \mathrm{H}_{13} \mathrm{NO}_{2}$ & Yellow & 0.58 \\
\hline
\end{tabular}

\section{Disc-diffusion method}

The disc diffusion method $[14,15]$ was used to determine the antibacterial activity of azomethine using a 6-mm disc were prepared from Whatman's filter paper no.1. Azomethine solutions of varying concentrations ranging from 125, 250 and $500 \mu \mathrm{g} / \mathrm{mL}$ were prepared. Nutrient agar was prepared, sterilized and used as the growth medium for the culture of microorganisms; $20 \mathrm{~mL}$ of the sterilized medium was poured into each sterilized petri dish, covered and allowed to solidify. Thereafter, the 16-h old broth cultures of the specified microorganisms were used for disc diffusion studies [13].The sample, control and standard treated discs were air-dried at room temperature, to remove any residual solvent which might interfere with the determination, sterilized and inoculated. These plates were initially placed at low temperature for $1 \mathrm{~h}$ to allow the maximum diffusion of compounds from the test disc into the agar plate and later incubated at $37^{\circ} \mathrm{C}$ for $24 \mathrm{~h}$ in case bacteria [16], after which the zone of inhibition will be easily observed.

\section{Statistical analysis}

Each experimental value is expressed as the mean \pm standard error mean $(n=3)$. Statistical analysis was performed using GraphPad Prism 5.0 and data analyzed using one-way analysis of variance (ANOVA) for comparison between groups followed by Dunnett's multiple comparison test at a significant level of $p<0.05$.

\section{RESULTS}

\section{Chemistry}

UV-Visible, IR, NMR and mass spectral data 
supported the structures of all newly synthesized compounds. The spectral data for the newly synthesized compounds are as follows.

N-(3-phenylallylidene)benzamine

(SB-1): Yield: $70 \%$; mp: 98-100 ${ }^{\circ} \mathrm{C}$; Anal. Calcd. for $\mathrm{C}_{15} \mathrm{H}_{13} \mathrm{~N}$ : C, 86.92; H, 6.32; N, $6.76 \%$. Found C, 86.89; H, 6.28; N, $6.66 \%$; IR $\left(\mathrm{KBr}, \mathrm{cm}^{-1}\right): 3085$ (=C-H stretching of aromatic ring), $3039(=\mathrm{C}-\mathrm{H}$ stretching of alkenyl group), $1600 \quad(-\mathrm{C}=\mathrm{N}$ stretching azomethine group), 1540-1600 (C=Cstretching of aromatic ring), 1320 (C-N, stretching of azomethine group); ${ }^{1} \mathrm{H}-\mathrm{NMR}$ (500.1 $\left.\mathrm{MHz} \mathrm{CDCl}_{3}-d, \delta / \mathrm{ppm}\right): 5.76(1 \mathrm{H}, t, J=9.5 \mathrm{~Hz}, \mathrm{H}-$ 2'), $6.52\left(1 \mathrm{H}, d, J=12 \mathrm{~Hz}, \mathrm{H}-3^{\prime}\right), 7.23-7.60$ (10H, $m$, phenyl), $8.24\left(1 \mathrm{H}, d, J=7.8 \mathrm{~Hz}, \mathrm{H}-1^{\prime}\right)$; ${ }^{13} \mathrm{C}-\mathrm{NMR}\left(100 \mathrm{MHz}, \mathrm{CDCl}_{3}, \delta / \mathrm{ppm}\right): 120.2\left(\mathrm{C}_{2^{\prime}}\right)$, $123.4 \quad\left(C_{3} \& C_{5}\right), 126.3\left(C_{5^{\prime}} \& C_{9^{\prime}}\right), 127.5\left(C_{1}\right)$, $128.4\left(\mathrm{C}_{7^{\prime}}\right), 129.2\left(\mathrm{C}_{6^{\prime}} \& \mathrm{C}_{8^{\prime}}\right), 131.1\left(\mathrm{C}_{2} \& \mathrm{C}_{6}\right)$, $135.8\left(\mathrm{C}_{4^{\prime}}\right), 139.2\left(\mathrm{C}_{3^{\prime}}\right), 150.4\left(\mathrm{C}_{4}\right), 164.3\left(\mathrm{C}_{1^{\prime}}\right)$; MS $\left(\mathrm{m} / \mathrm{z}\right.$, (relative abundance, \%)): $207\left(\mathrm{M}^{+}\right.$ 18.9), 107, 102, 77, 53, 51, 130 (BP, 100); UVVis $(\mathrm{MeOH})\left(\lambda_{\max } / \mathrm{nm}\right): 353$.

\section{4-(3-Phenylallylideneamino)benzaldehyde}

(SB-2): Yield: $79 \%$; mp: 130-134 ${ }^{\circ} \mathrm{C}$; Anal. Calcd. for $\mathrm{C}_{16} \mathrm{H}_{13} \mathrm{NO}$ : C, 81.68; H, 5.57; N, 5.95 \%. Found C, 81.56; H, 5.48; N, $5.87 \%$; IR (KBr, $\left.\mathrm{cm}^{-1}\right): 3050$ (=C-H stretching of aromatic ring), 3038 (=C-H stretching of alkenyl group), 2720, 2820 ( $\mathrm{C}-\mathrm{H}$ stretching of aldehyde group), 1725 $(\mathrm{C}=\mathrm{O}$ Stretching of aldehyde group), $1658(-\mathrm{C}=\mathrm{N}$ stretching azomethine group), 1540-1620 (C=Cstretching of aromatic ring), 1320 (C-N, stretching of azomethine group); ${ }^{1} \mathrm{H}-\mathrm{NMR}$ (500.1 $\left.\mathrm{MHz} \mathrm{CDCl}_{3}-d, \delta / \mathrm{ppm}\right): 5.81(1 \mathrm{H}, \mathrm{t}, J=9.5 \mathrm{~Hz}, \mathrm{H}-$ 2'), $6.63(1 \mathrm{H}, \mathrm{d}, J=12 \mathrm{~Hz}, \mathrm{H}-3$ '), $7.5-7.9(10 \mathrm{H}$, $m$, phenyl), $8.32(1 \mathrm{H}, \mathrm{d}, J=7.8 \mathrm{~Hz}, \mathrm{H}-1$ '), 9.94 (s, $1 \mathrm{H}$, aldehyde); ${ }^{13} \mathrm{C}-\mathrm{NMR}\left(100 \mathrm{MHz} \mathrm{CDCl}_{3}, \delta /\right.$ ppm): $120.5\left(\mathrm{C}_{2^{\prime}}\right), 123.8\left(\mathrm{C}_{3} \& \mathrm{C}_{5}\right), 126.2\left(\mathrm{C}_{5^{\prime}} \&\right.$ $\left.\mathrm{C}_{9^{\prime}}\right), 128.3\left(\mathrm{C}_{7^{\prime}}\right), 129.4\left(\mathrm{C}_{6^{\prime}} \& \mathrm{C}_{8^{\prime}}\right), 131.8\left(\mathrm{C}_{2} \& \mathrm{C}_{6}\right)$, $135.4\left(\mathrm{C}_{4^{\prime}}\right), 136.3\left(\mathrm{C}_{1}\right), 139.6\left(\mathrm{C}_{3^{\prime}}\right), 155.2\left(\mathrm{C}_{4}\right)$, $164.6\left(\mathrm{C}_{1^{\prime}}\right), 192.4(-\mathrm{CHO}) ; \mathrm{MS}(\mathrm{m} / \mathrm{z}$, (relative abundance, \%)): $235\left(\mathrm{M}^{+}, 20.4\right), 158,105,102$, 77, 53, 51 (BP, 100); UV-Vis $(\mathrm{MeOH})\left(\lambda_{\max } / \mathrm{nm}\right)$ : 307.

4-(3-Phenylallylideneamino)phenol (SB-3): Yield: $80 \%$; mp: 120-130 $\mathrm{C}$; Anal. Calcd. for $\mathrm{C}_{15} \mathrm{H}_{13} \mathrm{NO}$ : C, 80.69; $\mathrm{H}, 5.87 ; \mathrm{N}, 6.27 \%$. Found C, 80.56; H, 5.78; N, $5.82 \%$; IR $\left(\mathrm{KBr}, \mathrm{cm}^{-1}\right)$ : 3640 (Broad, O-H Str), 3055 (=C-H stretching of aromatic ring), 3038 (=C-H stretching of alkenyl group), $1668 \quad(-\mathrm{C}=\mathrm{N}$ stretching azomethine group), $1540-1600$ ( $\mathrm{C}=\mathrm{C}$ - stretching of aromatic ring), 1320 (C-N, stretching of azomethine group); ${ }^{1} \mathrm{H}-\mathrm{NMR}$ (500.1 $\left.\mathrm{MHz}, \mathrm{CDCl}_{3}-d, \delta / \mathrm{ppm}\right)$ : $5.4\left(1 \mathrm{H}, s,-\mathrm{OH}, \mathrm{D}_{2} \mathrm{O}\right.$ exchangeable $), 5.72(1 \mathrm{H}, t$, $\left.J=9.5 \mathrm{~Hz}, \mathrm{H}-2^{\prime}\right), 6.59\left(1 \mathrm{H}, d, J=12 \mathrm{~Hz}, \mathrm{H}-3^{\prime}\right)$, $7.4-7.8(10 \mathrm{H}, m$, phenyl), $8.29(1 \mathrm{H}, d, J=7.8$
$\left.\mathrm{Hz}, \mathrm{H}-1^{\prime}\right) ;{ }^{13} \mathrm{C}$ NMR (100 MHz, $\left.\mathrm{CDCl}_{3}, \delta / \mathrm{ppm}\right)$ : $118.4\left(\mathrm{C}_{2} \& \quad \mathrm{C}_{6}\right), 120.3 \quad\left(\mathrm{C}_{2^{\prime}}\right), 123.9 \quad\left(\mathrm{C}_{3} \& \quad \mathrm{C}_{5}\right)$, $126.5\left(\mathrm{C}_{5^{\prime}} \& \mathrm{C}_{9^{\prime}}\right), 128.4\left(\mathrm{C}_{7^{\prime}}\right), 129.2\left(\mathrm{C}_{6^{\prime}} \& \mathrm{C}_{8^{\prime}}\right)$, $135.8\left(\mathrm{C}_{4^{\prime}}\right), 139.2\left(\mathrm{C}_{3^{\prime}}\right), 141.6\left(\mathrm{C}_{4}\right), 157.2\left(\mathrm{C}_{1}\right)$, $164.3\left(\mathrm{C}_{1^{\prime}}\right)$; MS $(\mathrm{m} / \mathrm{z}$, (relative abundance, \%)): $223\left(\mathrm{M}^{+}, 18.5\right), 146,130,102,93,77,53,51$ (BP, 100); UV-Vis $(\mathrm{MeOH})\left(\lambda_{\max } / \mathrm{nm}\right): 302$.

\section{4-Methoxy-N-(3-phenylallylidene)benzamine}

(SB-4): Yield: $87 \%$; mp: 140-143 ${ }^{\circ} \mathrm{C}$; Anal. Calcd. for $\mathrm{C}_{16} \mathrm{H}_{15} \mathrm{NO}$ : C, 80.98; H, 6.37; N, 5.90 \%. Found C, 80.96; H, 6.33; N, $4.89 \%$; 80.59; IR $\left(\mathrm{KBr}, \mathrm{cm}^{-1}\right): 3052$ (=C-H stretching of aromatic ring), 3037 (=C-H stretching of alkenyl group), 2934, 2876 (C-H, Str), $1658 \quad(-\mathrm{C}=\mathrm{N}$ stretching azomethine group), 1540-1620 ( $\mathrm{C}=\mathrm{C}$ - stretching of aromatic ring), 1320 (C-N, stretching of azomethine group), 1255 (C-O-C, Str); ${ }^{1} \mathrm{H}-\mathrm{NMR}$ (500.1 MHz, $\left.\mathrm{CDCl}_{3}-d, \delta / p p m\right): 3.73\left(3 \mathrm{H}, s, \mathrm{CH}_{3}\right)$, $5.74\left(1 \mathrm{H}, t, J=9.5 \mathrm{~Hz}, \mathrm{H}-2{ }^{\prime}\right), 6.56(1 \mathrm{H}, d, J=12$ $\mathrm{Hz}, \mathrm{H}-3$ '), $7.3-7.75(10 \mathrm{H}, m$, phenyl), $8.36(1 \mathrm{H}$, $d, J=7.8 \mathrm{~Hz}, \mathrm{H}-1$ '); ${ }^{13} \mathrm{C}-\mathrm{NMR}\left(100 \mathrm{MHz} \mathrm{CDCl}_{3}\right.$, $\delta / \mathrm{ppm}): 56.3\left(-\mathrm{CH}_{3}\right), 116.2\left(\mathrm{C}_{3} \& \mathrm{C}_{5}\right), 120.4$ $\left(\mathrm{C}_{2^{\prime}}\right), 123.7\left(\mathrm{C}_{3} \& \mathrm{C}_{5}\right), 126.2\left(\mathrm{C}_{5^{\prime}} \& \mathrm{C}_{9^{\prime}}\right), 126.9\left(\mathrm{C}_{7^{\prime}}\right)$, $128.1\left(\mathrm{C}_{6^{\prime}} \& \mathrm{C}_{8^{\prime}}\right), 135.8\left(\mathrm{C}_{4^{\prime}}\right), 139.3\left(\mathrm{C}_{3^{\prime}}\right), 141.2$ $\left(\mathrm{C}_{4}\right), \quad 160.4\left(\mathrm{C}_{1}\right), 164.3$; $\mathrm{MS}(\mathrm{m} / \mathrm{z}$, (relative abundance, \%)): $237\left(\mathrm{M}^{+}, 19.0\right), 160,130,107$, 102, 77, 53, 51 (BP, 100); UV-Vis $(\mathrm{MeOH})$ $\left(\lambda_{\max } / \mathrm{nm}\right): 306$.

\section{4-Methoxy-2-(3-}

phenylallylideneamino)benzoic acid (SB-5): Yield: $80 \%$; mp: 150-154 ${ }^{\circ} \mathrm{C}$; Anal. Calcd. for $\mathrm{C}_{17} \mathrm{H}_{15} \mathrm{NO}_{3}: \mathrm{C}, 72.58 ; \mathrm{H}, 5.37 ; \mathrm{N}, 4.98 \%$. Found C, 72.52; H, 5.39; N, $4.92 \%$; IR $\left(\mathrm{KBr}, \mathrm{cm}^{-1}\right)$ : $3400-2500(\mathrm{OH}$ Str of $\mathrm{COOH}), 3057(=\mathrm{C}-\mathrm{H}$ stretching of aromatic ring), $3038 \quad(=\mathrm{C}-\mathrm{H}$ stretching of alkenyl group), 2500-2900 (O-H of $\mathrm{COOH}, \mathrm{Str}), 1720$ ( $\mathrm{C}=\mathrm{O}$ of $\mathrm{COOH}$ Str), 1658 ($\mathrm{C}=\mathrm{N}$ stretching azomethine group), 1540-1620 $(\mathrm{C}=\mathrm{C}$ - stretching of aromatic ring), 1280 (C-O stretching of $\mathrm{COOH}$ group), 1323 (C-N, stretching of azomethine group), 1250 (C-O-C stretching of ether group); ${ }^{1} \mathrm{H}-\mathrm{NMR}(500.1 \mathrm{MHz}$, $\left.\mathrm{CDCl}_{3}-d, \delta / \mathrm{ppm}\right): 3.74\left(3 \mathrm{H}, s, \mathrm{OCH}_{3}\right), 5.76(1 \mathrm{H}$, $\left.t, J=9.5 \mathrm{~Hz}, \mathrm{H}-2^{\prime}\right), 6.8\left(1 \mathrm{H}, d, J=12 \mathrm{~Hz}, \mathrm{H}-3^{\prime}\right)$, 7.64 - $7.92(10 \mathrm{H}, m$, phenyl), $8.38(1 \mathrm{H}, d, J=7.8$ $\left.\mathrm{Hz}, \mathrm{H}-1^{\prime}\right), 11.00(1 \mathrm{H}, \mathrm{s}, \mathrm{COOH}) ;{ }^{13} \mathrm{C}-\mathrm{NMR}(100$ $\left.\mathrm{MHz}, \mathrm{CDCl}_{3}, \delta / \mathrm{ppm}\right): 56.8\left(-\mathrm{CH}_{3}\right), 108.4\left(\mathrm{C}_{5}\right)$, $109.1\left(\mathrm{C}_{3}\right), 112.6\left(\mathrm{C}_{1}\right), 119.8\left(\mathrm{C}_{2^{\prime}}\right), 126.1\left(\mathrm{C}_{5^{\prime}} \&\right.$ $\left.\mathrm{C}_{9^{\prime}}\right), 127.2\left(\mathrm{C}_{7^{\prime}}\right), 128.6\left(\mathrm{C}_{6^{\prime}} \& \mathrm{C}_{8^{\prime}}\right), 132.4\left(\mathrm{C}_{2}\right)$, $135.7\left(\mathrm{C}_{4^{\prime}}\right), 138.3\left(\mathrm{C}_{3^{\prime}}\right), 150.5\left(\mathrm{C}_{4}\right), 163.2\left(\mathrm{C}_{1^{\prime}}\right)$, $167.8\left(\mathrm{C}_{6}\right), 169.6(-\mathrm{COOH}) ; \mathrm{MS}(\mathrm{m} / \mathrm{z}$, (relative abundance, \%)): $281\left(\mathrm{M}^{+}, 20.7\right), 204,151,130$, $102,77,53,51$ (BP, 100); UV-Vis $(\mathrm{MeOH})$ $\left(\lambda_{\max } / \mathrm{nm}\right): 338$.

4-(3-Phenylallylideneamino)benzoic acid (SB6): Yield: $86 \%$; mp: 130-140 ${ }^{\circ} \mathrm{C}$; Anal. Calcd. for $\mathrm{C}_{16} \mathrm{H}_{13} \mathrm{NO}_{2}$ : C, 76.42; H, $5.29 \mathrm{~N}, 5.52 \%$. Found 
Table 2: Minimum inhibitory concentration (MIC) of $\beta$-phenyl acrolein derivatives, SB-1 to SB-6

\begin{tabular}{|c|c|c|c|c|}
\hline \multirow{3}{*}{ Compound } & \multicolumn{4}{|c|}{ Minimum inhibitory concentration (MIC, $\mu \mathrm{g} / \mathrm{mL}$ ) } \\
\hline & \multicolumn{2}{|c|}{ Gram positive bacteria } & \multicolumn{2}{|c|}{ Gram negative bacteria } \\
\hline & B. subtilis & S. aureus & $P$. aeruginosa & K. pneumoniae \\
\hline SB-1 & 250 & 62.5 & 250 & 250 \\
\hline SB-2 & 125 & 62.5 & 125 & 250 \\
\hline SB-3 & 62.5 & 62.5 & 250 & 250 \\
\hline SB-4 & 125 & 62.5 & 62.5 & 250 \\
\hline SB-5 & 125 & 62.5 & 62.5 & 62.5 \\
\hline SB-6 & 125 & 62.5 & 62.5 & 62.5 \\
\hline
\end{tabular}

Table 3: Zone of inhibition of $\beta$-phenyl acrolein derivatives, SB-1 to SB-6

\begin{tabular}{lcccc}
\hline \multirow{2}{*}{ Compound } & \multicolumn{4}{c}{ Zone of inhibition $(\mathbf{m m}, \mathbf{2 5 0} \mu \mathrm{g} / \mathrm{mL})$} \\
\cline { 2 - 5 } & \multicolumn{2}{c}{ Gram positive bacteria } & \multicolumn{2}{c}{ Gram negative bacteria } \\
\cline { 2 - 5 } & B. subtilis & S. aureus & P. aeruginosa & K. pneumoniae \\
\hline SB-1 & $8.5 \pm 0.28$ & $9.9 \pm 0.57^{*}$ & $8.6 \pm 0.33$ & $7.8 \pm 0.17$ \\
SB-2 & $10.1 \pm 0.72$ & $10.8 \pm 0.92^{*}$ & $7.3 \pm 0.33$ & $7.3 \pm 0.67$ \\
SB-3 & $12.0 \pm 0.57^{*}$ & $10.0 \pm 1.00^{*}$ & $8.3 \pm 0.33$ & $7.3 \pm 0.33$ \\
SB-4 & $9.6 \pm 0.33$ & $10.6 \pm 0.33^{*}$ & $10.3 \pm 0.33^{*}$ & $6.3 \pm 0.33$ \\
SB-5 & $10.3 \pm 0.33$ & $11.3 \pm 0.33^{*}$ & $10.6 \pm 0.33^{*}$ & $12.0+0.10^{*}$ \\
SB-6 & $9.6 \pm 0.33$ & $10.3 \pm 0.88^{*}$ & $11.6 \pm 0.88^{*}$ & $12.3 \pm 0.57^{*}$ \\
Gentamicin & $12.3 \pm 0.33$ & $9.3 \pm 0.57$ & $10.0 \pm 0.57$ & $12.6 \pm 0.67$ \\
\hline
\end{tabular}

${ }^{*} p<0.05$, compared to Gentamycin (one-way ANOVA followed by Dunnett's multiple comparison test $(p<0.05$.); values are mean $\pm \operatorname{SEM}(n=3)$

C, 72.51; H, 5.32N, $4.88 \%$; IR $\left(\mathrm{KBr} \mathrm{cm}^{-1}\right): 3052$ (=C-H stretching of aromatic ring), $3036(=\mathrm{C}-\mathrm{H}$ stretching of alkenyl group), 2926, 2872 (C-H stretching of methyl group), $1668 \quad(-\mathrm{C}=\mathrm{N}$ stretching azomethine group), 1540-1600 ( $\mathrm{C}=\mathrm{C}$ stretching of aromatic ring), 1320 (C-N, stretching of azomethine group); ${ }^{1} \mathrm{H}-\mathrm{NMR}$ (500.1 $\left.\mathrm{MHz}, \mathrm{CDCl}_{3}-d, \delta / \mathrm{ppm}\right): 5.85(1 \mathrm{H}, t, J=9.5 \mathrm{~Hz}, \mathrm{H}-$ 2'), $6.76\left(1 \mathrm{H}, d, J=12 \mathrm{~Hz}, \mathrm{H}-3^{\prime}\right), 7.64-7.92$ $\left(10 \mathrm{H}, m\right.$,phenyl), $8.41\left(1 \mathrm{H}, d, J=7.8 \mathrm{~Hz}, \mathrm{H}-1^{\prime}\right)$, $11.00(1 \mathrm{H}, \quad s, \mathrm{COOH}) ;{ }^{13} \mathrm{C}-\mathrm{NMR}(100 \mathrm{MHz}$, $\mathrm{CDCl} 3, \delta / \mathrm{ppm}): 120.4\left(\mathrm{C}_{2}\right), 123.5\left(\mathrm{C}_{3} \& \mathrm{C}_{5}\right)$, $126.7\left(\mathrm{C}_{5} \& \mathrm{C}_{9}\right), 127.4\left(\mathrm{C}_{7^{\prime}}\right), 128.4\left(\mathrm{C}_{6} \& \mathrm{C}_{8^{\prime}}\right)$, $129.2\left(\mathrm{C}_{1}\right), 131.2\left(\mathrm{C}_{2} \& \mathrm{C}_{6}\right), 135.9\left(\mathrm{C}_{4^{\prime}}\right), 139.4$ $\left(\mathrm{C}_{3^{\prime}}\right), 155.4\left(\mathrm{C}_{4}\right), 164.8\left(\mathrm{C}_{1^{\prime}}\right), 169.6(-\mathrm{COOH}) ; \mathrm{MS}$ $\left(\mathrm{m} / \mathrm{z}\right.$, (relative abundance, \%)): $251\left(\mathrm{M}^{+}, 32.6\right)$, 174, 130, 121, 102, 77, 53, 51 (BP, 100); UV-Vis $(\mathrm{MeOH})\left(\lambda_{\max } / \mathrm{nm}\right): 307$.

\section{Antibacterial activity}

The newly synthesized $\beta$-phenyl acrolein derivatives were tested for their antibacterial potential against $B$. subtilis and $S$. aureus, $K$. pneumoniae, and $P$. aeruginosa, using gentamycin as standard. The results are given in Table 2 and Table 3.

\section{DISCUSSION}

Equimolar concentration of $\beta$-phenyl acrolein and substituted aromatic amines in the presence of basic catalyst, triethylamine resulted in formation of azomethine derivatives of $\beta$-phenyl acrolein moiety shown in Figure 1.

The $\lambda_{\max }$ for the newly synthesized azomethines was found to be in range from $300-440 \mathrm{~nm}$. The IR stretch at around $1650-1680 \mathrm{~cm}^{-1}$ showed the $\mathrm{C}=\mathrm{N}$ bond formation. The formation of azomethines was identified by the presence of triplet between 5.7 to $5.8 \mathrm{ppm}$, in proton NMR spectra. All other aliphatic and aromatic protons were observed within the expected regions. The novel compounds were further confirmed by their characteristic mass fragment spectra. The mass fragment pattern of compound SB-4 given in Figure 2, displayed parent ion peak at 237, base peak at 51 , and different fragment peaks at 160 , 130, 107, 102, 77, and 53.

Similarly, all the new compounds were characterized. This part confirmed the synthesis of a series of six new azomethines derivatives of $\beta$-phenyl acrolein.

The antibacterial potential of newly synthesized molecules was estimated by tube dilution and disc diffusion method; using Gram positive bacteria (Bacillus subtilis and Staphylococcus aureus) and Gram negative bacteria (Klebsiella pneumoniae and Pseudomonas aeruginosa). Tube dilution method depends upon the inhibition of growth of a microbial culture in a uniform solution of antibiotic in a fluid medium that is favorable to its rapid growth in the absence of the antibiotic [17]. In this method minimum inhibitory 
<smiles>O=C/C=C/c1ccccc1</smiles>

Beta phenyl acrolein<smiles>[R]c1cc([R])c(N)cc1[R]</smiles>

Aromatic amines
$\underset{\mathrm{TEA}, 60-70^{\circ} \mathrm{C}}{\stackrel{\text { Ethanol }}{\longrightarrow}}$<smiles>[R]c1cc([R])c(N=CC=Cc2ccccc2)cc1[R]</smiles>

Azomethines

Where,

$$
\begin{array}{llll}
\mathrm{R} 1=-\mathrm{H}, & \mathrm{R} 2=-\mathrm{H}, & \mathrm{R} 3=-\mathrm{H}, & \text { for SB-1 } \\
\mathrm{R} 2=-\mathrm{CHO}, & \mathrm{R} 2=-\mathrm{H}, & \mathrm{R} 3=-\mathrm{H}, & \text { for SB-2 } \\
\mathrm{R} 1=-\mathrm{OH}, & \mathrm{R} 2=-\mathrm{H}, & \mathrm{R} 3=-\mathrm{H}, & \text { for SB-3 } \\
\mathrm{R} 1=-\mathrm{OCH} H_{3}, & \mathrm{R} 2=-\mathrm{H}, & \mathrm{R} 3=-\mathrm{H}, & \text { for SB-4 } \\
\mathrm{R} 1=-\mathrm{H}, & \mathrm{R} 2=-\mathrm{OCH}_{3}, \mathrm{R} 3=-\mathrm{COOH}, & \text { for SB-5 } \\
\mathrm{R} 1=-\mathrm{COOH}, & \mathrm{R} 2=-\mathrm{H}, & \mathrm{R} 3=-\mathrm{H}, & \text { for SB-6 }
\end{array}
$$

Figure 1: Scheme for synthesis of azomethine derivatives of $\beta$-phenyl acrolein moiety

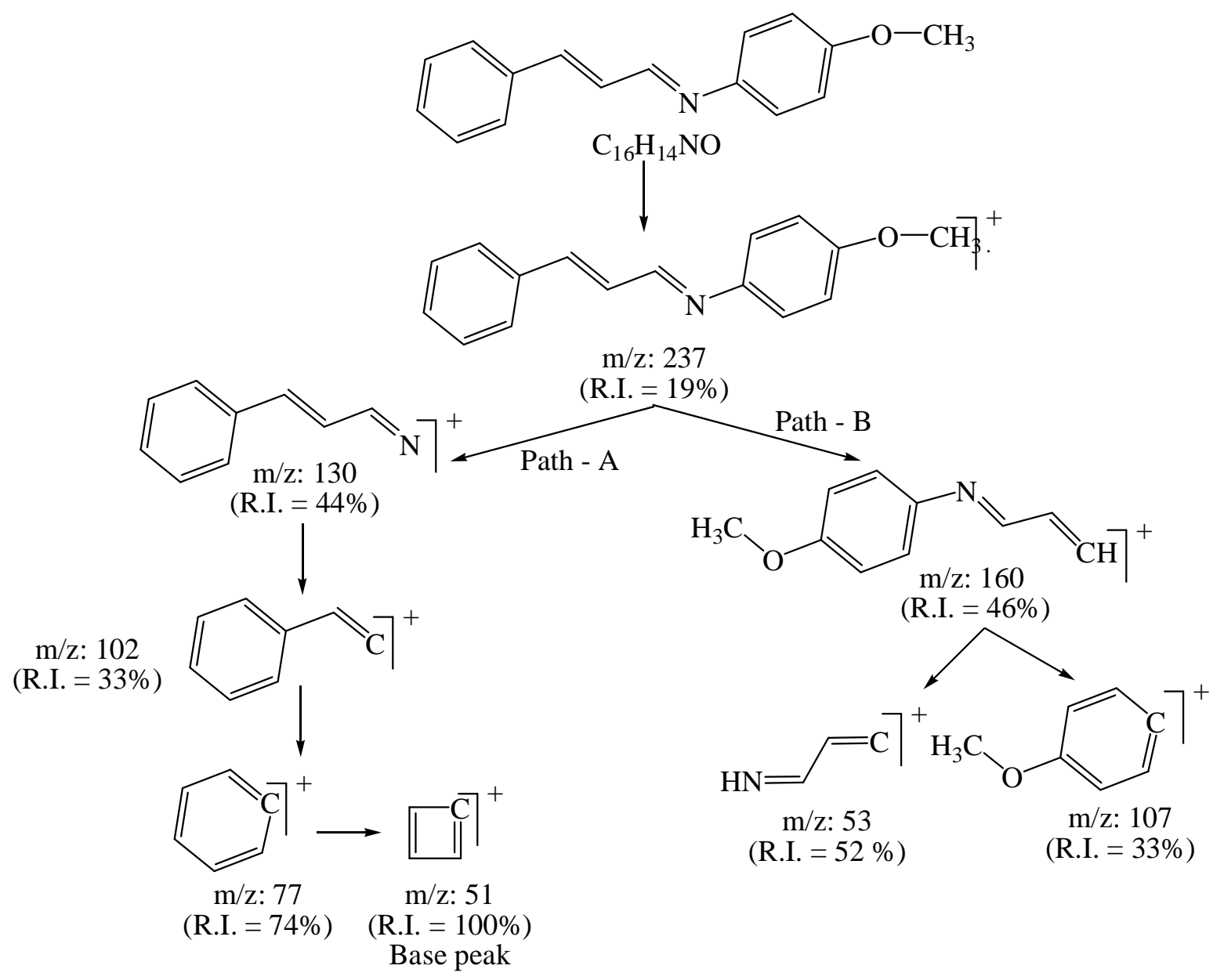

Figure 2: Mass fragmentation pattern of SB-4

concentration of the test compounds was determined. Gentamycin was used as a standard drug [18].

As per the minimum inhibitory concentration study results given in Table 2 and Table 3, among all synthesized compounds, SB-5 and SB-6 displayed highest MIC value of $62.5 \mu \mathrm{g} / \mathrm{ml}$. The zone of inhibition experiment, revealed that at MIC of $250 \mu \mathrm{g} / \mathrm{ml}$, all compounds SB-1 to SB6 showed significant antibacterial activity $(p<$ 0.05). SB-5 and SB-6 were active against $S$. aureus, $P$. aeruginosa and $K$. pneumoniae; SB-3 was active against $B$. subtilis and $S$. aureus, SB4 was active against $P$. aeruginosa and $S$. aureus. SB-1 and SB-2 were active against $S$. aureus. The antimicrobial results proved that all 
synthesized azomethines of $\beta$-phenyl acrolein moiety possess significant antibiotic potential.

\section{CONCLUSION}

$\beta$-Phenyl acrolein derivatives have been successfully synthesized and appear to be a novel and important class of antibacterial agents against Gram-positive and Gram-negative bacteria including $S$. aureus, $P$. aeruginosa, and $K$. pneumonia. The synthetic route and antibacterial potential of the compounds may be useful in guiding future efforts to synthesize new compounds with improved antibacterial activity.

\section{ACKNOWLEDGEMENT}

The authors are thankful to AIMST University, Malaysia, for providing funds via a grant, and also for the facilities to carry out the research. The authors are also thankful to UiTM, Malaysia for providing technical support in generating the analytical data.

\section{CONFLICT OF INTEREST}

No conflict of interest associated with this work.

\section{AUTHORS' CONTRIBUTION}

We declare that this work was done by the author(s) named in this article and all liabilities pertaining to claims relating to the content of this article will be borne by them. Sridevi Chigurupati, Neeraj kumar Fuloria and Ravichandran Veerasamy carried out the synthetic work, Syed adnan ali Shah, Shivkanya Fuloria and Sundram Karupia performed the analytical work while Appala raju Nemala, Lim jun Yi and Ang xiang Ilan carried carried out the antibacterial activities. All authors approved the manuscript for publication.

\section{REFERENCES}

1. Yossa N, Patel J, Dumitru M, Patricia M, Charles M. Antibacterial activity of Cinnamaldehyde and Sporan Against Escherichia Coli 0157:H7 and Salmonella. J Food Process Preserv 2012; 38: 749-757.

2. Cabello CM, Bair WB 3rd, Sarah Lamore D, Stephanie, L, Alexandra Bause S, Sara A, Georg Wondrak, T. The Cinnamon-derived Michael Acceptor Cinnamic Aldehyde Impairs Melanoma Cell Proliferation, Invasiveness, and Tumor Growth. Free Radic Biol Med 2009; 46: 220-231.

3. Karl-Georg F, Franz-Josef $H$, Johannes $P$, Wilhelm $P$, Dietmar S, Kurt B, Dorothea G, Horst S. Flavors and Fragrances in Ullmann's Encyclopedia of Industrial
Chemistry, Wiley-VCH, Verlag GmbH \& Co. 2002; $p$ 141.

4. Amin RM, Abdel-Kader NS, El-Ansary AL. Microplate assay for screening the antibacterial activity of Schiff bases derived from substituted benzopyran-4-one. SpectrochimActa. Part A 2012; 9: 517-525.

5. Neelakantan $M$, Esakkiammal $M$, Mariappan $S$, Dharmaraja J, Jeyakumar T. Synthesis, Characterization and Biocidal Activities of Some Schiff Base Metal Complexes. Indian J Pharm Sci 2010; 72: 216-222.

6. Fugu MB, Ndahi NP, Paul BB, Mustapha AN. Synthesis, characterization, and antimicrobial studies of some vanillin schiff base metal (II) complexes. J Chem Pharm Res 2013; 5: 22-28.

7. Song WJ, Cheng JP, Jiang DH, Guo L, Cai MF, Yang $H B$, Lin, QY. Synthesis, interaction with DNA and antiproliferative activities of two novel Cu(II) complexes with Schiff base of benzimidazole. Spectrochimica acta. Part A 2014; 121: 70-76.

8. Liang C, Xia J, Lei D, Li X, Yao Q, Gao J. Synthesis, in vitro and in vivo antitumor activity of symmetrical bisSchiff base derivatives of isatin. Euro $J$ Med Chem 2014; 74: 742-750.

9. Zoubi WA. Biological Activities of Schiff Bases and Their Complexes: A Review of Recent Works. Int J Org Chem 2013; 3: 73-95.

10. Amirnasar M, Mahmoudkhani AH, Gorji A, Dehghanpour S, Bijanzadeh HR. Cobalt(II), nickel(II), and zinc(II) complexes with bidentate $N, N^{\prime}$-bis(phenylcinnamaldehyde)-1,2-diiminoethane Schiff base: synthesis and structures. Polyhedron 2002; 21: 2733-2742.

11. Juan S, Yong Y, Gui-Hua S, Zhi-Bo Y Hai-Liang Z. Synthesis, molecular modeling and structural characterization of vanillin derivatives as antimicrobial agents. J Mol Struct 2013; 1039: 214-218.

12. Chigurupati $S$. Designing new vanillin schiff bases and their antibacterial Studies. J Med Bioeng 2015; 4: 363366.

13. Sridevi $\mathrm{CH}$, Balaji $K$, Naidu $A$, Sudhakaran $R$. Antimicrobial evaluation and synthesis of some phenylpyrazolo benzothiazolo quinoxaline derivatives. $J$ Chem 2009; 6: 866-870.

14. Delignette-Muller ML, Flandrois, JP. An accurate diffusion method for determining bacterial sensitivity to antibiotics. J Antimicrob Chemother 1994; 34: 73-81.

15. Jessy EM, Thirugnana Sambanthan A, Alex J, Sridevi $\mathrm{CH}$, Srinivasan KK. Synthesis and biological evaluation of some novel quinazolones. Indian J Pharm Sci 2007; 69: 476-478.

16. Sridevi $\mathrm{CH}$, Kannan MM, Abhinayani G, Sravya $N$. Designing and Biological Evaluation of new benzimidazole compounds. Chem Sci Trans 2013; 2: 922-926.

17. Cappucino JG.; Sherman N. Microbiology - A Laboratory Mannual,4th edn, Addison Wesley Longmann, New York, USA; 1999; $p 45$. 
Chigurupati et al

18. Bala S, Sharma N, Kajal A, Kamboj S. Design, Synthesis, Characterization, and Computational Studies on Benzamide Substituted Mannich Bases as Novel,
Potential Antibacterial Agents. Sci World J 2014; 2014: 1-9. 